\title{
Clinical and hormonal features of women with polycystic ovary syndrome living in rural and urban areas
}

\author{
Krzysztof Katulski', Adam Czyzyk², Natalia Podkowa', Agnieszka Podfigurna', Natalia Ignaszak², \\ Katarzyna Paczkowska², Sylwia Slawek², Dariusz Szpurek³ ${ }^{3}$ Blazej Meczekalski' \\ ${ }^{1}$ Department of Gynecological Endocrinology, Poznan University of Medical Sciences, Poznan, Poland \\ 2 Students Scientific Society of the Department of Gynecological Endocrinology, Poznan University of Medical Sciences, \\ Poznan, Poland \\ ${ }^{3}$ Division of Gynecological Surgery, Poznan University of Medical Sciences, Poznan, Poland
}

Katulski K, Czyzyk A, Podkowa N, Podfigurna A, Ignaszak N, Paczkowska K, Slawek S, Szpurek D, Meczekalski B. Clinical and hormonal features of women with polycystic ovary syndrome living in rural and urban areas. Ann Agric Environ Med. 2017; 24(3): 522-526. doi: 10.5604/12321966.1227642

\begin{abstract}
Introduction. Polycystic ovary syndrome (PCOS) is one of the most common endocrinopathies among women at reproductive age, but its pathology remains unknown. From epidemiological studies it is known that endogenous, mainly genetic and exogenous, environmental factors are of importance.

Objective. The aim of the study was to compare the phenotype of women diagnosed with PCOS from urban and rural areas of Poland. According to the knowledge of the authors, this is first such study.

Materials and method. The retrospective study included 3,877 PCOS patients: 2511 women living in cities and 1,366 village inhabitants, aged between $18-45$ years. Clinical data, including medical history, body mass, height and hirsutism severity was obtained from each patient. Hormones were also tested in each patient: follicle stimulating hormone, luteinizing hormone, prolactin, estradiol [E2], testosterone, dehydroepiandrosterone sulphate [DHEAS], thyroid stimulating hormone, free thyroxin, insulin [INS], 17 hydroxyprogesterone, cortisol [CORT]) and metabolic (75g oral glucose tolerance test, Chol - total cholesterol, HDL-C - high density lipoprotein cholesterol, LDL-C low density lipoprotein cholesterol, and the TG (triglicerides) profile.

Results. PCOS women from urban areas had a higher mean serum concentration of E2 in comparison to the inhabitants of rural areas. Women from cities had a lower mean level of DHEAS, CORT, and INS measured in the morning than rural residents. Insulin-resistance, using homeostasis model assessment, was more pronounced among women from villages. The prevalence of menstrual disorders, in general, was higher in PCOS women living in rural comparing to urban areas. Conclusions. The clinical and biochemical indices differed significantly between women diagnosed with PCOS living in cities and villages. In general in Poland, the PCOS phenotype is more severe in women living in rural areas. This study shows that different living conditions significantly affect the PCOS phenotype.
\end{abstract}

Key words

Polycystic ovary syndrome, environment, pathogenesis

\section{INTRODUCTION}

Polycystic ovary syndrome (PCOS) is one of the most common endocrine disorders among women, occurring with the prevalence between $6-15 \%$, depending on diagnostic criteria $[1,2,3]$. The disease is characterized by hyperandrogenaemia, chronic anovulation and polycystic ovarian morphology in ultrasound [1]. Infertility due to anovulatory cycles is also a clinically important problem in PCOS patients $[2,3]$. The most often observed feature is hyperandrogenism, which manifests as hirsutism, acne, alopecia and seborrhea [4]. The clinical picture of PCOS consists also of insulin resistance (IR), obesity, impaired glucose tolerance (IGT), arterial hypertension (AT), diabetes mellitus (DM) and increased prevalence of metabolic syndrome [4]. Some authors also report increased cardiovascular risk [1].

Consensus workshops, sponsored by the NIH/NIHCD, ESHRE/ASRM and Androgen Excess \& PCOS Society,

Address for correspondence: Blazej Meczekalski, Department of Gynecological Endocrinology, Poznan University of Medical Sciences, Poznan, Poland

E-mail: blazejmeczekalski@yahoo.com

Received: 06 January 2014; accepted: 28 May 2014; first published: December 2016 developed three different diagnostic criteria. However it seems that most clinicians use the Rotterdam criteria from 2003 to recognize PCOS [5].

Because of the disease complexity, PCOS etiology is still unclear. Both prenatal and postnatal factors are postulated to be important for its pathogenesis. Many studies have been performed to explain the pathogenesis of the disease, and results indicate genetic and environmental factors to be elusive $[1,6,7,8,9]$. Genetic susceptibility seems to induce the disease, but extrinsic factors certainly modify the clinical picture of PCOS and influences its' variety. Clinical trials showed a hereditary pattern of PCOS in some families; however, even in the same family phenotypes vary widely, which suggests that not only genetic factors are involved in PCOS pathology [6]. Known environmental risk factors playing a role in PCOS pathogenesis are: in utero exposure of the foetus to androgen excess, tobacco and alcohol use, socioeconomic status related with parental and personal education level, premature pubarche, peripubertal stress, nutrition, physical activity, obesity, medications and environment pollution $[7,8,9]$. Considering the disease pathogenesis and risk factors, special attention must be paid for the influence 
of ethnic origin, geographical location and culture on PCOS expression [1].

\section{OBJECTIVE}

Taking into account studies postulating the geographical influence on PCOS symptoms in women from different regions of the world, this study was undertaken to investigate whether environment can also influence the clinical features among women of the same ethnicity, but living in different areas of the same country. Comparison of the clinical and biochemical features of patients diagnosed with PCOS was made between inhabitants of cities and villages in Poland.

\section{MATERIALS AND METHOD}

The retrospective study comprised 2,511 women living in cities and 1,366 village inhabitants aged between 18-45 years, hospitalized in the Department of Gynecological Endocrinology in Poznan, Poland, in 2007-2012. The patients from both groups were matched according to age and body mass index (BMI). Medical history was taken from every patient, including the age of menarche, menstrual irregularity, length of menstrual cycles, gynecological history, presence of other diseases, use of contraceptives or hormonal replacement therapy, signs of hyperandrogenism, former hospitalizations and surgeries. Patients were asked about their place of residence, and using administrative definitions, were divided to inhabitants of villages and cities.

Gynecological and ultrasound examination were performed. Patients younger than 18 and older than 45 years were excluded from the study. Other excluding criteria were congenital adrenal hyperplasia (measurement of serum 17-hydroxyprogesterone), Cushing's syndrome (morning and afternoon serum cortisol levels, $1 \mathrm{mg}$ dexamethasone test), hyperprolactinaemia (prolactin levels above $30 \mathrm{ng} / \mathrm{ml}$ ), thyroid dysfunction (thyreotropin and free thyroxin seerum levels).

Definition and symptoms of PCOS. To diagnose PCOS, the Rotterdam criteria were used [5]. Two of three criteria had to be fulfilled: 1) oligo- and/or anovulation; 2) clinical and/or biochemical hyperandrogenism; 3) polycystic ovarian morphology in ultrasound. Oligoovulation was defined as the absence of menstruation for more than 35 days, or the presence of less than nine menses in a year; anovulation was diagnosed in the case of lack of spontaneous menstruation for at least 3 months. Hyperandrogenaemia was defined as a serum testosterone level higher than $0.8 \mathrm{ng} / \mathrm{ml}$, and/or DHEAS concentration above $9.5 \mathrm{mmol} / \mathrm{l}$. Clinical hyperandrogenism was diagnosed as hirsutism, defined as modified-Ferriman-Gallwey score 8 or higher (examination performed by an experienced specialist in gynecological endocrinology). The patient was diagnosed as overweight if BMI was between $25-29,99 \mathrm{~kg} / \mathrm{m}^{2}$, and obese if the BMI was $30 \mathrm{~kg} / \mathrm{m}^{2}$ or higher. The presence of IR was confirmed using homeostasis model assessment (HOMA). Incorrect insulin function was defined as HOMA-IR above 2.5. Systolic and diastolic blood pressure (sBP and $\mathrm{dBP}$ ) was measured after at least 15 minutes of rest.
Laboratory tests. In women with oligomenorrhea, blood samples were taken on the tenth day of menstrual cycle; in the case of secondary amenorrhea, the blood was taken on a random day of the cycle after overnight fast. Serum levels of follicle-stimulating hormone (FSH), luteinizing hormone (LH), prolactin (PRL), estradiol (E2), testosterone (T), thyroid-stimulating hormone (TSH), free thyroxine (fT4), dehydroepiandrosterone sulfate (DHEAS), 17-hydroxyprogesterone (17OHP), cortisol (CORT), were taken in the morning and in the afternoon, and insulin (INS) and fasting plasma glucose (FPG) measured. Serum concentrations of FSH, LH, PRL, E2, T, DHEAS, INS, TSH, fT4, 17OHP were measured using enzymoimmunoassay method (EIA). Serum glucose and lipoprotein fractions [total cholesterol (Chol), HDL cholesterol (HDL-C), LDL cholesterol (LDL-C), triglicerydes (TG)] levels were quantified by enzymatic method. OGTT was performed after dosing with $75 \mathrm{~g}$ of glucose.

Statistical analysis. All parameters were described as mean \pm standard deviation (SD). If the data did not fulfill the criteria of normal distribution, the non-parametric Mann-Whitney test was used; otherwise, the Welch corrected test was introduced. As statistically significant, a two-tailed p-value of less than 0.05 was established. A confidence interval of $95 \%$ was assumed. Data analyses were performed by Statistica 10.0 (StatSoft 2011).

\section{RESULTS}

Women living in urban areas had significantly higher mean concentrations of estradiol $(67.37 \pm 52.34 \mathrm{pg} / \mathrm{ml})$ in comparison to women living in villages $(63.89 \pm 46.35 \mathrm{pg} / \mathrm{ml}$; $\mathrm{p}=0.0351)$. City inhabitants had a significantly lower mean serum level of DHEAS and CORT measured in the morning $(8.20 \pm 3.32 \mathrm{umol} / 1,512.4 \pm 170.3 \mathrm{nmol} / \mathrm{l}$, respectively) than rural residents $(8.63 \pm 3.37 \mathrm{umol} / \mathrm{l}, 537.9 \pm 171.4 \mathrm{nmol} / \mathrm{l}$; $\mathrm{p}=0.0034, \mathrm{p}=0.0080$ respectively). Urban dwellers also had lower mean serum concentration of INS than village inhabitants $(11.10 \pm 9.00$ vs. $11.49 \pm 8.56 \mu \mathrm{U} / \mathrm{ml} ; \mathrm{p}=0.0132)$. A significantly lower SBP was also observed in patients from cities than country dwellers $(115 \pm 13$ vs. $117 \pm 14 \mathrm{mmHg}$; $\mathrm{p}=0.0353)$. Women living in cities had significantly lower HOMA-IR (2.42 \pm 2.33$)$ in comparison to women living in villages $(2.52 \pm 2.29 ; \mathrm{p}=0.0327)$. The prevalence of menstrual disorders, such as oligomenorrhea and amenorrhea, varied significantly ( $\mathrm{p}=0.0127, \mathrm{p}=0.0323$, respectively), between women living in urban and rural areas. City inhabitants suffered more often from oligomenorrhea and less often from amenorrhea, compared with village occupants; the prevalence of menstrual disturbances was $65.7 \%$ vs. $61.6 \%$ and $45.5 \%$ vs. $49.2 \%$, relatively. The characteristics of both clinical and hormonal features are shown in Tables 1 and 2, and differences shown in Figures 1-6.

\section{DISCUSSION}

To the best of the authors' knowledge, the presented data is the first such extensive analysis of phenotype of PCOS in a Polish population. It is also the first to compare the clinical and biochemical features of PCOS between women living in 
Table 1. Clinical features of urban and rural inhabitants

\begin{tabular}{lccc}
\hline & $\begin{array}{c}\text { URBAN } \\
\mathrm{n}=2511\end{array}$ & $\begin{array}{c}\text { RURAL } \\
\mathrm{n}=1366\end{array}$ & $\mathrm{p}$-value \\
\hline Age [years] & $25 \pm 5$ & $24 \pm 4$ & 0.1117 \\
\hline BMI [kg/m²] & $26.1 \pm 6.9$ & $26.5 \pm 6.7$ & 0.0567 \\
\hline Underweight [\%] & 4.8 & 3.4 & 0.0657 \\
\hline Normal weight [\%] & 47.7 & 46.4 & 0.4850 \\
\hline Overweight [\%] & 20.6 & 21.2 & 0.6884 \\
\hline Obesity [\%] & 26.9 & 29.0 & 0.2126 \\
\hline Blood pressure [mmHg] & \multicolumn{3}{c}{} \\
\hline Systolic BP & $\mathbf{1 1 5} \pm \mathbf{1 3}$ & $\mathbf{1 1 7} \pm \mathbf{1 4}$ & $\mathbf{0 . 0 3 5 3}$ \\
\hline Diastolic BP & $73 \pm 9$ & $74 \pm 10$ & 0.7748 \\
\hline Ferriman-Gallwey score [points] & $19 \pm 7$ & $20 \pm 7$ & 0.3730 \\
\hline$<8$ points [\%] & 3.9 & 3.7 & 0.8605 \\
\hline$\geq 8$ points [\%] & 96.1 & 96.3 & 0.8605 \\
\hline Menstrual disorders [\%] & \multicolumn{3}{c}{} \\
\hline oligomenorrhea & $\mathbf{6 5 . 7}$ & $\mathbf{6 1 . 6}$ & $\mathbf{0 . 0 1 2 7}$ \\
\hline amenorrhea & $\mathbf{4 5 . 5}$ & $\mathbf{4 9 . 2}$ & $\mathbf{0 . 0 3 2 3}$ \\
\hline dysmenorrhea & 2.5 & 1.7 & 0.1328 \\
\hline Eumenorrhea & 13.7 & 12.0 & 0.1571 \\
HOMA-IR & $\mathbf{2 . 4 2 \pm 2 . 3 3}$ & $\mathbf{2 . 5 2 \pm 2 . 2 9}$ & $\mathbf{0 . 0 3 2 7}$ \\
\hline Insulin resistance [\%] & 83.3 & 83.6 & 0.8604 \\
\hline
\end{tabular}

BMI - body mass index; BP - blood pressure; HOMA-IR - homeostasis assessment model of insulin resistance.

Table 2. Hormonal and biochemical characteristics of the city and village inhabitants

\begin{tabular}{|c|c|c|c|}
\hline & $\begin{array}{l}\text { URBAN } \\
\mathrm{n}=2511\end{array}$ & $\begin{array}{l}\text { RURAL } \\
n=1366\end{array}$ & $p$-value \\
\hline FSH [IU/I] & $6.09 \pm 1.94$ & $6.14 \pm 1.93$ & 0.4431 \\
\hline LH [IU/I] & $12.49 \pm 6.45$ & $12.66 \pm 6.22$ & 0.1761 \\
\hline PRL [ng/ml] & $16.81 \pm 11.35$ & $16.49 \pm 9.85$ & 0.6159 \\
\hline E2 [pg/ml] & $67.37 \pm 52.34$ & $63.89 \pm 46.35$ & 0.0351 \\
\hline $\mathrm{T}[\mathrm{ng} / \mathrm{ml}]$ & $0.700 \pm 0.299$ & $0.704 \pm 0.313$ & 0.7657 \\
\hline DHEAS [ $\mu \mathrm{mol} / \mathrm{l}]$ & $8.20 \pm 3.32$ & $8.63 \pm 3.37$ & 0.0034 \\
\hline TSH [IU/l] & $2.78 \pm 1.80$ & $2.87 \pm 2.13$ & 0.9219 \\
\hline fT4 [ng/ml] & $1.31 \pm 0.26$ & $1.32 \pm 0.29$ & 0.3862 \\
\hline INS [uU/ml] & $11.10 \pm 9.00$ & $11.49 \pm 8.56$ & 0.0132 \\
\hline 17OHP [nmol/l] & $1.864 \pm 0.778$ & $1.979 \pm 0.876$ & 0.1731 \\
\hline CORT7 [nmol/l] & $512.4 \pm 170.3$ & $537.9 \pm 171.4$ & 0.0080 \\
\hline $\begin{array}{l}\text { CORT4 [nmol/l] } \\
\text { FPG [mg/dl] }\end{array}$ & $\begin{array}{c}239.2 \pm 122.4 \\
84.7 \pm 13.3\end{array}$ & $\begin{array}{c}237.1 \pm 121.5 \\
85.2 \pm 16.2\end{array}$ & $\begin{array}{l}0.7240 \\
0.8959\end{array}$ \\
\hline Glc in $0^{\prime}$ of OGTT [mg/dl] & $91.9 \pm 14.3$ & $92.4 \pm 12.0$ & 0.2760 \\
\hline Glc in $60^{\prime}$ of OGTT [mg/dl] & $160.1 \pm 38.2$ & $157.5 \pm 36.3$ & 0.5786 \\
\hline $\mathrm{Glc}$ in $120^{\prime}$ of OGTT $[\mathrm{mg} / \mathrm{dl}]$ & $126.2 \pm 35.1$ & $125.1 \pm 33.4$ & 0.6359 \\
\hline Chol [mg/dl] & $191.5 \pm 36.0$ & $193.4 \pm 37.4$ & 0.3552 \\
\hline $\mathrm{HDL}-\mathrm{C}[\mathrm{mg} / \mathrm{dl}]$ & $53.6 \pm 13.1$ & $52.5 \pm 12.6$ & 0.1393 \\
\hline LDL-C [mg/dl] & $115.0 \pm 32.5$ & $116.7 \pm 33.3$ & 0.3869 \\
\hline $\mathrm{TG}[\mathrm{mg} / \mathrm{dl}]$ & $114.6 \pm 65.0$ & $117.8 \pm 79.0$ & 0.4303 \\
\hline
\end{tabular}

FSH - follicle stimulating hormone; LH - luteinizing hormone; PRL - prolactin; E2 - estradiol; T testosterone; DHEAS - dehydroepiandrosterone sulphate; TSH - thyroid stimulating hormone; fT4 - free thyroxin; INS - insulin; 17OHP - 17 hydroxyprogesterone; CORT7 - cortisol at 07:00 a.m. CORT4 - cortisol at 04:00 p.m.; FPG - fasting plasma glucose; Glc - glucose; OGTT - 75g oral glucose tolerance test; Chol - total cholesterol; HDL-C - high density lipoprotein cholesterol; LDL-C low density lipoprotein cholesterol; TG - triglicerides

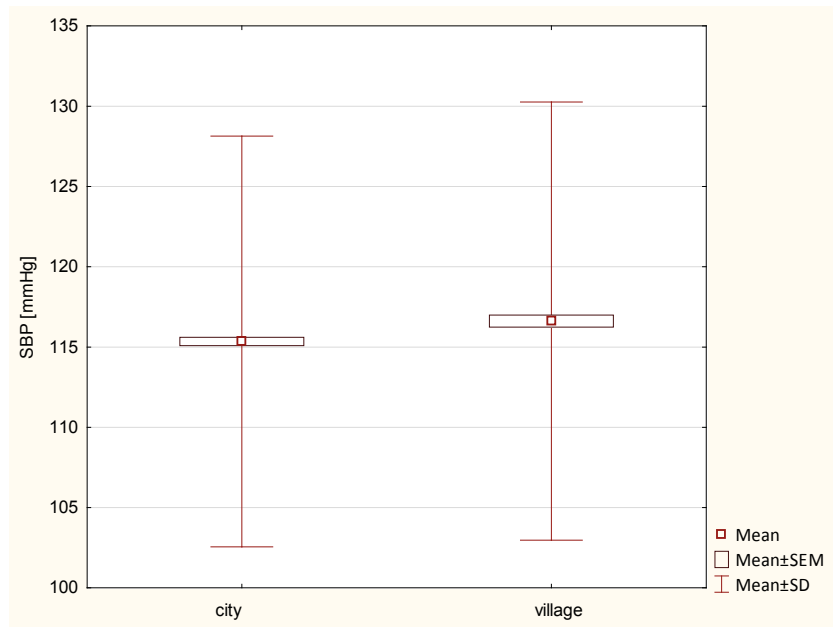

Figure 1. Mean, standard error of measurement (SEM) and standard deviation (SD) of systolic blood pressure values among PCOS city and village inhabitants

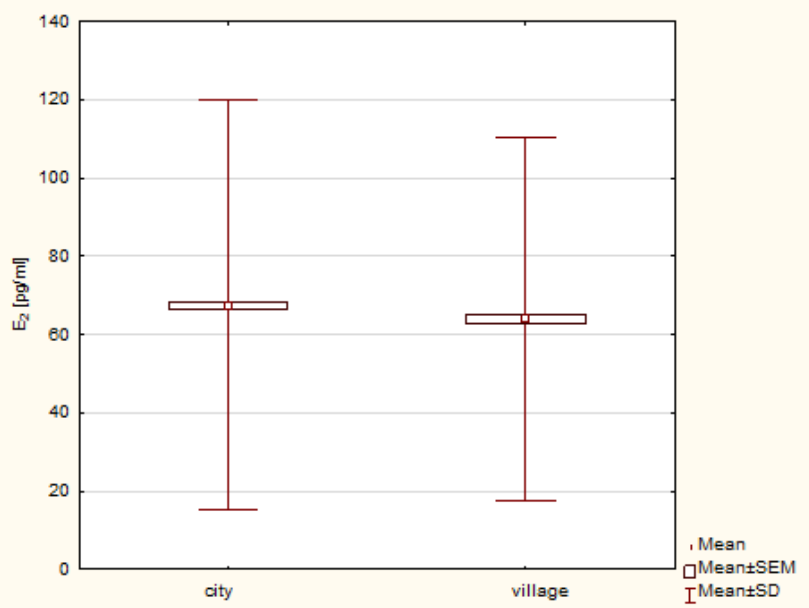

Figure 2. Mean, standard error of measurement (SEM) and standard deviation (SD) of serum E2 concentration among PCOS city and village inhabitants

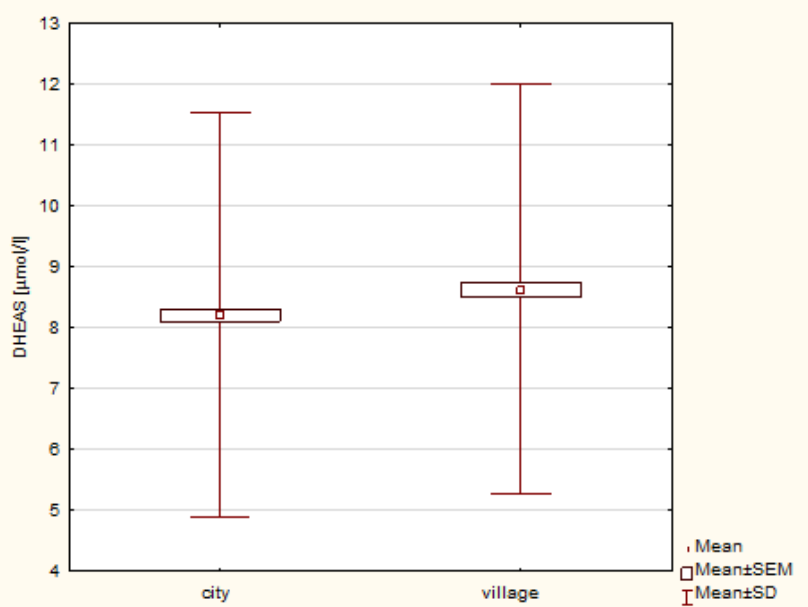

Figure 3. Mean, standard error of measurement (SEM) and standard deviation (SD) of serum DHEAS concentration among PCOS city and village inhabitants 


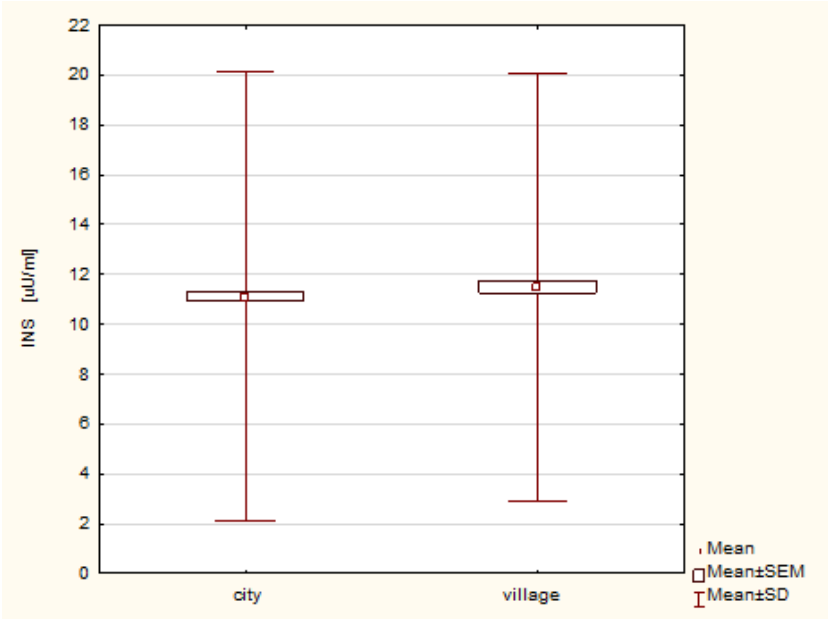

Figure 4. Mean, standard error of measurement (SEM) and standard deviation (SD) of serum INS concentration among PCOS city and village inhabitants

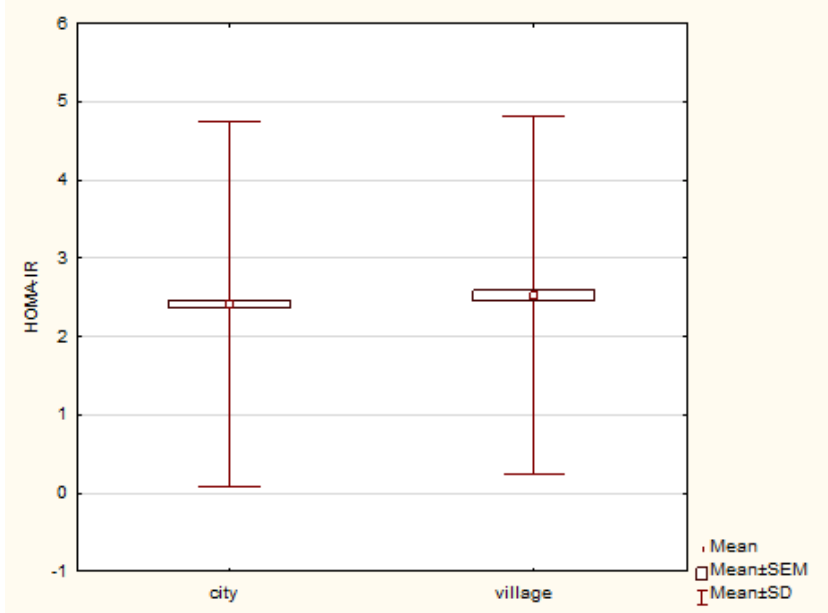

Figure 5. Mean, standard error of measurement (SEM) and standard deviation (SD) of HOMA-IR values among PCOS city and village inhabitants

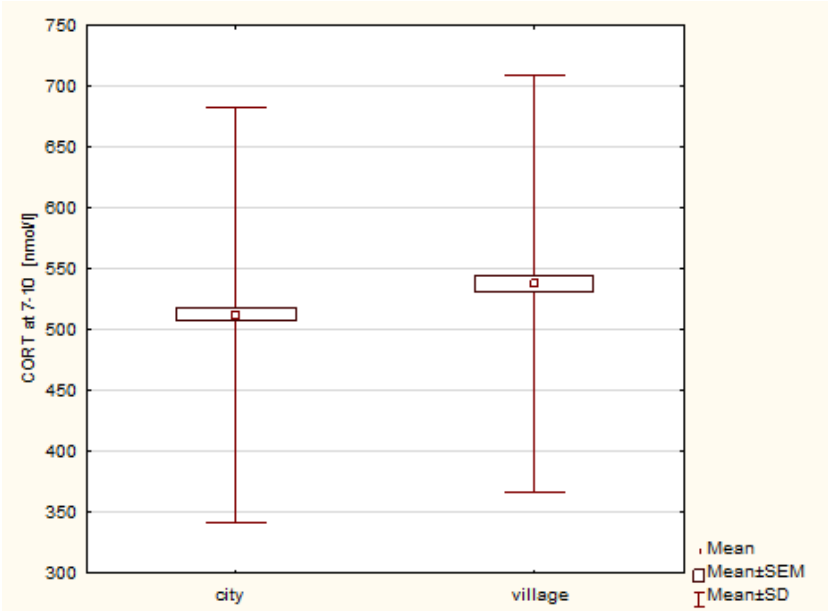

Figure 6. Mean, standard error of measurement (SEM) and standard deviation (SD) of serum CORT concentration measured at 7-10 in the morning among PCOS city and village inhabitants urban and rural areas. This analysis brings to the forefront the issue of environmental factors in pathogenesis of PCOS.

The most important finding of this study is that, generally, a more severe clinical picture was found in the inhabitants of villages. This refers particularly to menstrual pattern (increased prevalence of amenorrhea), lower mean levels of estradiol and increased mean serum concentrations of DHEAS, INS and morning cortisol. Also, insulin resistance was more pronounced in women living in rural areas, and interestingly, the same was revealed for sBP. The observed differences are very difficult to explain, especially if one takes into consideration the number of possible factors affecting the PCOS phenotype. The groups were age and BMI matched to exclude the influence of those known important factors on the clinical picture of the studied disease. Even so, higher insulin levels and HOMA-IR were found in patients living in small communities. Theoretically, this fact could be an explanation for the slightly (but statistically significant) worse clinical and hormonal parameters of the inhabitants of villages [10]. Insulin-resistance is known to be associated with a more severe PCOS phenotype. Moreover, insulin sensitivity is known to be related to many environmental factors, such as physical activity, diet, and alcohol consumption, among others [11]. The failed could not find any studies focusing on the problem of the different prevalence of insulin-resistance in urban and rural areas; however, there are few analyzing the occurrence of metabolic syndrome. Some studies have reported the higher prevalence of metabolic syndrome among urban population compared to their counterparts in rural areas; however, information on the distribution of metabolic syndrome and its combinations by urban/rural in middle income countries, such as Poland, are limited $[12,13]$. In general, living in urban areas is associated with the prevalence of metabolic syndrome (central adiposity, lipids, lipoproteins, and blood pressure measures), whereas the rural environment has a protective effect. On the other hand, in those studies, the groups were not BMI matched, which can be a key factor in determining the indices of insulin sensitivity.

Another important and possibly elusive factor is the higher cortisol levels in PCOS women from rural areas. Steroid hormones play a central role in the regulation of both ovarian function and body composition. Increased peripheral cortisol metabolism has been reported to be associated with PCOS pathogenesis in a manner that is independent of BMI $[14,15]$. It is hypothesized that a compensatory up-regulation of the hypothalamic-pituitary-adrenal (HPA) axis is responsible for the excess of adrenal androgens [dehydroepiandrosterone (DHEA) and DHEA sulphate (DHEAS)], and their urinary excretion metabolites in PCOS. Therefore, the increased cortisol stimulation in the presented study group from villages could, theoretically, be the reason for the aggravated metabolic and hormonal picture of the disease in these individuals.

Stress that evokes uncertainty and anxiety, or where the environmental stimulus exceeds available coping resources and induces negative emotions, will lead to increased levels of cortisol as a result of the activation of hypothalamicpitutary-adrenal axis (HPA-axis) [16]. It could therefore be speculated that unfavourable environmental stressors in women from villages could cause increased HPA activity which, in turn, interferes with metabolic and hormonal indices in PCOS. Increased HPA stimulation could also be causative for increased sBP [16]. 
Taken together, it is very difficult to explain the observed differences between women diagnosed with PCOS living in different environments. The presented study has discussed only some from a possible huge number of factors which could influence the course of the disease. It was impossible to assess the exposition to air, water and food pollution, the role of the use of fertilizers in villages, known to act as endocrine disruptors, and even different patterns of physical activity. The list of such potential factors is endless; however, bearing in mind the scale of the study, it is considered that the differences cannot be neglected. This indicates a big field of expertise for environmental medicine, and for the first time, brings to the fore the importance of impact of the patient's immediate environment on the course of PCOS. On the other hand, this study indicates that women living in rural areas require more attention, especially in the context of metabolic health.

\section{CONCLUSIONS}

In the light of intrauterine programming theory, it can be supposed that in the pathology of PCOS, intrinsic factors play a major role in triggering the disease. It is proposed that increased androgenic activity of foetal gonads is causative for the adult PCOS phenotype. However, this study also indicates the important role of environmental factors. Interesingly, animal models have shown a large variability of PCOS-like phenotypes ensuing from foetal androgen exposure $[2,17]$, and the same has been observed in humans. Such diverse phenotypes after the same intrauterine exposure suggest a strong influence of extrinsic factors. Among them, diet, physical activity, alcohol and nicotine consumption, as well as the place of residence, are important. Therefore, it is reasonable to link the observed differences in PCOS phenotype living in villages and cities with the environmental, life-style and pollution differences in those two areas.

\section{REFERENCES}

1. Fauser BC, Tarlatzis BC, Rebar RW et al. Consensus on women's health aspects of polycystic ovary syndrome (PCOS): the Amsterdam ESHRE/ ASRM-Sponsored 3rd PCOS Consensus Workshop Group. Fertil Steril. 2012; 97(1): 28-38.

2. Baird DT, Balen A, Escobar-Morreale HF. Health and fertility in World Health Organization group 2 anovulatory women. Hum Reprod Update. 2012; 18(5): 586-99.
3. Kozakowski J, Zgliczyński W. Body composition, glucose metabolism markers and serum androgens - association in women with polycystic ovary syndrome. Endokrynol Pol. 2013; 64(2): 94-100.

4. Nawrocka-Rutkowska J, Ciećwież S, Marciniak A, Brodowska A, Wiśniewska B, Kotlega D, Starczewski A. Insulin resistance assessment in patients with polycystic ovary syndrome using different diagnostic criteria--impact of metformin treatment. Ann Agric Environ Med. 2013; 20(3): 528-32.

5. Rotterdam ESHRE/ASRM-Sponsored PCOS consensus workshop group. Revised 2003 consensus on diagnostic criteria and long-term health risks related to polycystic ovary syndrome (PCOS). Hum Reprod. 2004; 19(1): 41-52.

6. Diamanti-Kandarakis E,Piperi CH, Argyrakopoulou G, Spina J, Papanastasiou L, Bergiele A, Panidis D. Polycystic Ovary Syndrome: The influence of environmental and genetic factors. Hormones 2006; 5(1): 17-34.

7. Brzozowska M, Karowicz-Bilińska A.The role of vitamin D deficiency in the etiology of polycystic ovary syndrome disorders. Ginekol Pol. 2013; 84(6): 456-60.

8. Xita N, Tsatsoulis A. Review: fetal programming of polycystic ovary syndrome by androgen excess: evidence from experimental, clinical, and genetic association studies. J Clin Endocrinol Metab. 2006; 91(5): 1660-6.

9. Katulski K, Czyzyk A, Meczekalski B. The controversies in the diagnosis of polycystic ovary syndrome. Pol Merkur Lek. 2012 Jul; 33(193): 32-7.

10. Vaggopoulos V, Trakakis E, Chrelias C, Panagopoulos P, Basios G, Makridima S, Sioulas VD, Simeonides G, Labos G, Boutati E, Kassanos D. Comparing classic and newer phenotypes in Greek PCOS women: the prevalence of Metabolic Syndrome and their association with Insulin Resistance. J Endocrinol Invest. 2012 Nov 27.

11. Cai H, Huang J, Xu G, Yang Z, Liu M, Mi Y, Liu W, Wang H, Qian D. Prevalence and determinants of metabolic syndrome among women in Chinese rural areas. PLoS One. 2012; 7(5): e36936.

12. Gu D, Reynolds K, Wu X, Chen J, Duan X, Reynolds RF, Whelton PK, He J. Prevalence of the metabolic syndrome and overweight among adults in China. Lancet. 2005; 365: 1398-1405. doi: 10.1016/S01406736(05)66375-1.

13. Das M, Pal S, Ghosh A. Rural urban differences of cardiovascular disease risk factors in adult Asian Indians. Am J Hum Biol. 2008 JulAug; 20(4): 440-5.

14. Vassiliadi DA, Barber TM, Hughes BA, McCarthy MI, Wass JA, Franks S, Nightingale P, Tomlinson JW, Arlt W, Stewart PM. Increased 5 alphareductase activity and adrenocortical drive in women with polycystic ovary syndrome. J Clin Endocrinol Metab. 2009; 94(9): 3558-66.

15. Valkenburg O, Uitterlinden AG, Themmen AP, de Jong FH, Hofman A, Fauser BC, Laven JS. Hum Reprod. Genetic polymorphisms of the glucocorticoid receptor may affect the phenotype of women with anovulatory polycystic ovary syndrome. 2011; 26(10): 2902-11.

16. Huisman HW, van Rooyen JM, Malan NT, Eloff FC, Malan L, Laubscher PJ, Schutte AE. Prolactin, testosterone and cortisol as possible markers of changes in cardiovascular function associated with urbanization. J Hum Hypertens. 2002 Dec; 16(12): 829-35.

17. Panidis D, Tziomalos K, Chatzis P. Association between menstrual cycle irregularities and endocrine and metabolic characteristics of the polycystic ovary syndrome. Eur J Endocrinol. 2013; 168(2): 145-52. 